\title{
Randomized controlled trials: who fails run-in?
}

\author{
Judy R Rees ${ }^{1 *}$, Leila A Mott ${ }^{1}$, Elizabeth L Barry ${ }^{1}$, John A Baron ${ }^{1,4}$, Janet L Peacock ${ }^{2,3}$ \\ From 3rd International Clinical Trials Methodology Conference \\ Glasgow, UK. 16-17 November 2015
}

\section{Background}

Early identification of enrollees at risk of poor adherence and run-in failure (RIF) may present opportunities to increase trial efficiency and generalizability.

\section{Methods}

We conducted a factorial-design randomized, controlled trial of calcium and vitamin D to prevent colorectal adenoma recurrence. At the enrolment interview, study coordinators at 11 centers collected demographic and medical information and participants' beliefs about the study tablets. Participants also completed two self-administered questionnaires (SAQ) before a three-month single-blinded placebo run-in. Eligible participants were then randomized to calcium, vitamin D, both or neither; women electing to take calcium were randomized to vitamin $\mathrm{D}$ or placebo. A priori, we considered three subgroups: men $(\mathrm{N}=1606)$ and women $(\mathrm{N}=301)$ in the full factorial randomization and women in the 2 -arm randomization $(\mathrm{N}=666)$.

\section{Results}

Overall, 314 of 2,573 (12\%) enrollees potentially eligible for randomization failed run-in due to poor adherence (took $<80 \%$ tablets) or refusal to participate. In multivariable models in the largest subgroup (males), RIF was associated with younger age (adjusted odds ratio per 5 years 0.85 ; 95\% CI 0.76-0.96), single marital status (1.67; 1.12-2.49), any missing data on the SAQs $(2.05 ; 1.46-2.86)$ study center $(\mathrm{p}<0.0001)$ and perceived toxicity report $(12.86$; 5.41-30.56). Across all three subgroups, the latter three factors were most consistently associated with RIF but other factors are described which vary by subgroup.

'Geisel School of Medicine at Dartmouth, Department of Epidemiology, Hanover, NH, USA

Full list of author information is available at the end of the article

\section{Conclusions}

The most consistent predictors of RIF were perceived toxicities, missing data on self-administered questionnaires, and study center. The latter two findings relate to study coordinator oversight, and present potential opportunities to improve adherence during run-in.

\section{Authors' details}

'Geisel School of Medicine at Dartmouth, Department of Epidemiology, Hanover, NH, USA. 'Division of Health and Social Care Research, King's College, London, UK. ${ }^{3} \mathrm{NIHR}$ Biomedical Research Centre at Guy's and St Thomas' NHS Foundation Trust and King's College, London, UK. 'University of North Carolina at Chapel Hill, NC, USA.

Published: 16 November 2015

doi:10.1186/1745-6215-16-S2-078

Cite this article as: Rees et al:: Randomized controlled trials: who fails run-in? Trials 2015 16(Suppl 2):O78.
Submit your next manuscript to BioMed Central and take full advantage of:

- Convenient online submission

- Thorough peer review

- No space constraints or color figure charges

- Immediate publication on acceptance

- Inclusion in PubMed, CAS, Scopus and Google Scholar

- Research which is freely available for redistribution
() Biomed Central 